\title{
Neuropsychological deficits in chronic fatigue syndrome: artifact or reality?
}

Chronic fatigue syndrome is an illness characterised by extreme fatigue of uncertain origin which has been present for at least six months. The fatigue should have a sudden onset and be severe enough to substantially reduce the patient's day to day activities. ${ }^{1}$ A number of other symptoms are associated with chronic fatigue syndrome including complaints of impaired memory, difficulty making decisions, poor attention, and reduced concentration. ${ }^{1}$ Many patients complain that it is these cognitive symptoms which cause them the greatest frustration and disability.

Over the past six years several researchers have conducted neuropsychological tests on patients with chronic fatigue syndrome in an attempt to objectively quantify the nature and extent of these cognitive difficulties. Despite the objective aims of these studies the pattern of results has been contradictory and often confusing. The aim of this review is to provide a comprehensive summary and discussion of the results from neuropsychological studies which have compared patients with chronic fatigue syndrome with controls or normative data, to provide both some clarity in the area and directions for future research.

Studies published since the Centre for Disease Control issued the first diagnostic criteria for chronic fatigue syndrome $^{2}$ were accessed through a search on the MEDLINE and PsycLIT databases. Only fully reported studies were reviewed, as we were unable to reliably assess the limited information presented in the abstracts. The results of the search are grouped into five neuropsychological categories; global intellectual functioning, receptive functioning (encompassing sensation and perception), mental activity (subgrouped into activity rate and attentional functions), memory, and cognition (which incorporates the higher mental functions of planning, organising, problem solving, and abstracting). ${ }^{3}$ We also examine the relations between cognitive impairment in chronic fatigue syndrome and both psychological and physical factors.

\section{Global intellectual functioning}

Studies which have measured overall intellectual functioning have found that there is no evidence of intellectual decline or a primary deficit in intellectual functioning in chronic fatigue syndrome. ${ }^{4-8}$ Comparisons of the scores of patients with chronic fatigue syndrome with normative data on the Wechsler adult intelligence scale-revised (WAIS-R) have shown that patients with chronic fatigue syndrome score within the normal range on both the full scale verbal and performance IQs. ${ }^{578}$ Similarly, no differences have been found between matched control groups and patients with chronic fatigue syndrome on the Shipley institute of living scale and the national adult reading test. 457

\section{Receptive functions}

There is no clear evidence of sensory or perceptual impairments in chronic fatigue syndrome. Two studies have directly examined the sensory functions in patients with chronic fatigue syndrome by measuring brain event related potentials (ERPs). ${ }^{89}$ No significant differences were found in brainstem, visual, and somatosensory ERPs between healthy controls and patients with chronic fatigue syndrome suggesting that the neural processing of sensory information by the patients is intact. With regard to perception, patients with chronic fatigue syndrome performed within the normal range on the Luria Nebraska visual functions scale, which measures visual and spatial perception. ${ }^{10}$ Perceptual abilities can also be inferred to some extent from results on other tests of general mental functioning and activity. For example, to perform effectively on the WAIS-R block design and object assembly tests, patients need adequate visuospatial conceptualisation. Patients with chronic fatigue syndrome perform well within the normal ranges and are comparable with matched controls on such tests. ${ }^{51112}$

\section{Mental activity}

\section{ACTIVITY RATE}

There is evidence that patients with chronic fatigue syndrome are slower to react to stimuli. This deficit seems to be related to delayed information processing, rather than impaired motor responses. Two studies found that whereas error rates on visual and auditory discrimination tasks in patients with chronic fatigue syndrome were similar to healthy controls, their reaction times were significantly slower. ${ }^{89}$ Patients with chronic fatigue syndrome also showed slower reaction times in two studies using tests of simple and serial reaction times, ${ }^{13} 14$ although one study found no difference between controls and patients with chronic fatigue syndrome on these tests. ${ }^{6}$ Patients with chronic fatigue syndrome performed within the normal range and were comparable with healthy controls on three measures of psychomotor performance. ${ }^{711}{ }^{15} \mathrm{On}$ an item recognition task designed to assess the effect of mental load on performance, Sandman et $a l^{10}$ reported that when compared with controls, mental scanning and encoding of information in patients with chronic fatigue syndrome was significantly delayed in response to an increase in memory load, but the psychomotor response was not.

\section{ATTENTIONAL ACTIVITIES}

Verbal attention in patients with chronic fatigue syndrome has been assessed with three tests: the digit span test, the serial sevens task, and the paced auditory serial addition test (PASAT). Digit span is a measure of both short term memory and attention. Eight studies have shown that performance on digit span in patients with chronic fatigue syndrome is comparable with both normative data and matched controls. ${ }^{611-17}$ On the other hand, one study reported that patients with chronic fatigue syndrome performed worse on serial sevens than would be expected from controls. ${ }^{15}$ However, the lack of clear diagnostic criteria, a control group, and standardised norms for the 
serial sevens task means that this isolated finding should be treated with caution. The PASAT, which is a complex task of sustained divided attention and is highly dependent on response speed, has been used in two studies. ${ }^{17} 18$ Both reported that patients with chronic fatigue syndrome performed worse than matched healthy controls. As patients with chronic fatigue syndrome perform well within the normal range on the digit span test, poorer performances on the PASAT may reflect slower information processing rather than a deficit in sustained verbal attention.

Six studies using a range of measures of visual attention and concentration have found that the performance of patients with chronic fatigue syndrome is within the normal range, and comparable with matched control groups. These measures include the trail making test, ${ }^{47111217}$ the embedded figures test, ${ }^{19}$ and the digit symbol subtest of the WAIS-R ${ }^{412}$ although this has been less consistent. ${ }^{1120}$ Three studies using cancellation tasks have reported deficits in visual attention but these results are hard to interpret due to the use of unstandardised tests ${ }^{13} 14$ and chronic fatigue syndrome samples which are either unclearly defined ${ }^{13}$ or do not necessarily meet standard diagnostic criteria. ${ }^{15}$ On the Stroop test-a visual attention task which includes an assessment of susceptibility to interference of irrelevant stimuli-patients with chronic fatigue syndrome, when compared with controls, consistently show slower reaction times on the interference task. ${ }^{12} 141920$

In summary, there is little objective evidence to suggest that patients with chronic fatigue syndrome have impaired motor responses, or ability to focus and sustain attention. Rather, the strongest and most consistent evidence of impaired mental activity is in delayed reaction times and slowed information processing. Slower processing seems to be particularly apparent on complex cognitive tasks which require divided attention over a lengthy period and in which interference or memory load is increased.

\section{Memory}

Two memory batteries, the California verbal learning test (CVLT) and the Wechsler memory scale (WMS) have been used for patients with chronic fatigue syndrome. The composite scores across studies on both of these measures suggest that memory in patients with chronic fatigue syndrome is generally intact. Findings from two studies reported CVLT scores within the normal range and no differences between controls and patients with chronic fatigue syndrome on any of the CVLT variables. ${ }^{121}$ However, DeLuca et $a l^{17}$ found that their chronic fatigue syndrome group had significantly more difficulty than controls on the acquisition component of the CVLT but not with storage and retrieval. They suggest that the difference in these findings may be a result of the more stringent selection criteria for their chronic fatigue syndrome sample and that this group's difficulty with acquisition may have reflected a slowness in processing information.

Studies have consistently reported WMS scores within the normal range..$^{5101216}$ Despite these normal composite scores, there do seem to be some conflicting differences between the scores of patients with chronic fatigue syndrome and controls on certain WMS subtests. ${ }^{671116}$ The lack of consistent findings on these subscales, and the fact that despite reported differences subscale scores are typically in the normal range, ${ }^{12}$ suggest that the differences may reflect variation in the samples rather than true memory deficits. With two exceptions, ${ }^{6}{ }^{18}$ the vast majority of studies have found no deficit in either verbal or visual free recall. $^{5611-17}$
Although free recall seems to be unimpaired, patients with chronic fatigue syndrome may be vulnerable to interference on memory tasks and have greater difficulty when speed of processing is integral to the memory task. Four studies using tests which depend on cued and contextual recall such as paired associates learning and memory for story ideas, have found that patients with chronic fatigue syndrome benefit less from cueing than healthy controls. $^{671016}$ Others have reported that patients with chronic fatigue syndrome are more sensitive than controls to proactive inhibition on memory tasks ${ }^{10}$ and are slower to retrieve general knowledge information during semantic memory tasks. ${ }^{1314}$ However, an almost equal number of studies have found no differences between patients with chronic fatigue syndrome and controls on paired associates ${ }^{51115}$ or semantic memory tests. ${ }^{710} \mathrm{~A}$ study published in this issue of the fournal assessed a wide range of memory functions in patients with chronic fatigue syndrome using a computerised battery of tests. ${ }^{22}$ Patients with chronic fatigue syndrome were impaired on memory tasks which required greater mental effort, such as producing self ordered responses from memory, and memorising semantically unrelated word pairs.

In summary, no consistent or severe memory impairment is evident from the performance of patients with chronic fatigue syndrome on neuropsychological tests. Patients' verbal and visual free recall is unimpaired, but some studies have found that they gain less benefit from cueing and context on memory tasks than healthy controls. This is an unusual finding which warrants further investigation, as most patients with cognitive deficits perform better on cued recall than free recall. ${ }^{6}$ Patients with chronic fatigue syndrome also seem to be more sensitive to interference and effort on memory tasks than would be expected from healthy controls. These memory impairments may reflect attentional rather than memory deficits. The way in which information is attended to and processed has a direct impact on the quality of the memory trace. ${ }^{23}$ In particular, slowed processing can limit elaboration of information during encoding. This would help to explain why patients with chronic fatigue syndrome have some difficulty on tasks that rely on more complex effort, elaboration, or organisation of material such as memorising lists of words that are not semantically associated, or ordering sequences of responses in memory. ${ }^{22}$

\section{Cognition}

Tests of cognition or higher mental functioning generally assess a broad range of functions. For instance, the standard version of the Wisconsin card sorting test ${ }^{24}$ requires normal memory and attention capacities, adequate motor skills, and the ability to abstract and categorise information in working memory. ${ }^{25}$ Patients with chronic fatigue syndrome perform both within the normal range and equal to healthy controls on this test, providing further evidence that there is no distinct global cognitive impairment in chronic fatigue syndrome. ${ }^{712}$ Performances of patients with chronic fatigue syndrome on other tests of concept formation - the similarities subtest of the WAIS$\mathrm{R}$, the symbol digit modalities test, the booklet category test, and the short category test-verify that both their verbal and non-verbal ability to conceptualise is unimpaired. ${ }^{411} 1718$ Similarly, patients with chronic fatigue syndrome have performed consistently well on tests which examine the ability to order, organise, and plan, such as the object assembly and block design tests (WAIS-R), and the Tower of London and Hanoi tests. ${ }^{561112}$ Grafman et $a l^{6}$ also examined reasoning ability through an activity in which patients had 20 questions to solve a particular 
problem. Patients with chronic fatigue syndrome performed as well as controls on this task, consistent with the weight of evidence which shows no impairment of higher cognitive functions.

\section{Psychological factors and cognitive deficits}

People with chronic fatigue syndrome have concurrent psychiatric disorders, usually depression, at a greater level than both the general population and people with other chronic illnesses ${ }^{2627}$ Like patients with chronic fatigue syndrome, patients with depression often complain of cognitive difficulties. Objective testing suggests that depressed patients often have an impairment in short term memory and information processing but this generally improves as the depression resolves. ${ }^{28-30}$ It is therefore important to identify how depression may contribute to the cognitive deficits reported in chronic fatigue syndrome. Using self report measures of depression, six separate studies of patients with chronic fatigue syndrome found no relation between depression scores and objective performance on neuropsychological tests. ${ }^{5614171822}$ However, methodological problems may account for these negative results. In two studies results were analysed by using a median split on their depression measure. ${ }^{14} 17$ As only about $26 \%$ of patients with chronic fatigue syndrome can be expected to have concurrent depression, ${ }^{26}$ using a median split is problematical. In addition, self reported depression scales are not always an accurate reflection of the presence of clinical depression in the context of illnesses such as chronic fatigue syndrome, because a number of somatic items included in the scales overlap with key symptoms of chronic fatigue syndrome. ${ }^{31}$ When diagnostic schedules for depression have been used, depression has been related to performance of patients with chronic fatigue syndrome on some short term memory tests. ${ }^{11}{ }^{15}$ Poorer performance on attention tasks and prolonged reaction times in chronic fatigue syndrome have been related to other psychological states, specifically heightened mental fatigue, emotional distress, and increased somatic complaints. 15192232

Other researchers have used matched groups of clinically depressed patients. Three out of four studies found no significant differences between chronic fatigue syndrome and depressed groups on a wide range of memory, mental activity, and cognitive scores. ${ }^{5} 1217$ Despite similarities in performance, patients with chronic fatigue syndrome reported significantly more subjective cognitive complaints ${ }^{517}$ than did the depressed patients. One study, using only specific memory measures, reported that whereas there were no differences between the groups on free recall, patients with chronic fatigue syndrome had greater difficulty than depressed patients on cued recall and proactive inhibition tasks. ${ }^{10}$

A consistent finding across studies is the discrepancy between objective performance and subjective reports of cognitive difficulty, with studies invariably reporting no relation between objective test results and subjective complaints. ${ }^{45819}$ Rather, subjective reports of impairment have been consistently related to higher levels of psychopathology, anxiety, depression, and somatic complaints. ${ }^{561315}$ Patients with chronic fatigue syndrome also report higher levels of fatigue and symptoms of stress during the testing procedure when compared with both healthy and psychiatric controls, although this is not evident from their performance. ${ }^{81232}$

In summary, depression, emotional distress, fatigue, and somatic ratings have all shown some relations to impaired performance in chronic fatigue syndrome. In addition, patients with chronic fatigue syndrome con- sistently rate themselves as more cognitively impaired, stressed by performance, and fatigued than both healthy controls and depressed patients, although there is no objective evidence to support these self ratings. As such, patients with chronic fatigue syndrome may have a bias for processing somatic or fatigue related information and increased attention or overconcern about somatic information, may affect attentional processes.

\section{Physical factors and cognitive deficits}

The relations between biological assays, MRI abnormalities, and ERPs to neuropsychological task performance in chronic fatigue syndrome have been explored in various studies. No studies have found a relation between biological assays and objective cognitive impairment. The assays investigated include the VP1 antigen, ${ }^{9}$ antibodies to the Epstein-Barr and Coxsackie viruses, ${ }^{7}$ enteroviral genomic RNA sequence in muscle, and the presence of abnormal mitochondria. ${ }^{14}$ Similarly, MRI abnormalities do not seem to be related to neuropsychological performance in chronic fatigue syndrome. ${ }^{5}$

Two ERP studies report conflicting results. The more rigorous study, which controlled for medication, time of day, and normal to corrected vision found no differences in patients with chronic fatigue syndrome and controls for ERPs recorded before, during, and after two to three hours of testing. ${ }^{8}$ The other study reported ERP abnormalities in one of their two auditory tasks and concluded that patients with chronic fatigue syndrome showed impaired attention and information processing. ${ }^{9}$

The neuropsychological performance of patients with chronic fatigue syndrome has also been compared with that of patients with multiple sclerosis. Although an initial study reported that both patients with multiple sclerosis and those with chronic fatigue syndrome performed worse than healthy controls on an attention task, ${ }^{18}$ when a depressed comparison was included in a later design, patients with chronic fatigue syndrome had subtle cognitive impairments that more closely resembled the depressed group than the multiple sclerosis group. ${ }^{17}$ Another study also found patients with chronic fatigue syndrome to have less severe cognitive impairment than patients with multiple sclerosis but higher depressive symptomatology. ${ }^{11}$ In addition, depression was related to neuropsychological performance in the chronic fatigue syndrome group but not in the multiple sclerosis group. Consequently, there is almost no evidence to date that organic factors contribute to the neuropsychological impairment in chronic fatigue syndrome.

\section{Conclusions and future research}

Despite the conflicting conclusions drawn by researchers, a pattern does seem to be evident as to the nature of neuropsychological impairment in chronic fatigue syndrome. The most consistent findings include slower reaction times, poorer performances on complex attentional and memory tasks, and, less consistently, a slowness in acquiring new information. A single deficit such as slower information processing may be responsible for all these positive findings. Overall, the impairments are slight and do not seem to have any effect on the higher cognitive functions of planning, organising, problem solving, and conceptualisation.

Some of the inconsistencies in the medical literature highlight the methodological difficulties that have hampered much of the research published to date. Many studies have relied on small sample sizes drawn predominately from hospital clinic settings and some have 
not used standard chronic fatigue syndrome diagnostic criteria. The pattern of scores for patients with chronic fatigue syndrome on neuropsychological tests is typically more widely spread than those of control groups and so the detection of any true differences is difficult without the use of larger samples than many of the studies have used. Furthermore, control for the time of day of testing, medication, and for type 1 error when making multiple comparisons have often been neglected in past studies.

The fact that only psychological rather than organic factors have shown a relation to neuropsychological deficits to date point to the key role of psychological factors in explaining neuropsychological symptoms in chronic fatigue syndrome. Patients with chronic fatigue syndrome consistently have higher self ratings of cognitive impairment but these deficits are unrelated to objective tests of cognitive performance. Future research on neuropsychological functioning in chronic fatigue syndrome should include psychological states as part of the assessment and preferably use psychiatric diagnostic groups as well as self report measures to stratify within chronic fatigue syndrome samples. ${ }^{1}$ An important focus in future studies should be to identify the underlying mechanisms involved, such as the role of the patient's personal illness beliefs in contributing to their subjective reports of cognitive difficulty. ${ }^{33}$ Somatic focus and overconcern about symptoms may play an important part in speed of information processing by competing for attentional resources. As chronic fatigue syndrome has a profoundly disabling effect on people's lives, an important omission in previous research has been the absence of data relating neuropsychological findings to daily functioning and the ability to work.

RONA MOSS-MORRIS

KEITH J PETRIE

ROBERT G LARGE ROBERT R KYDD

Department of Psychiatry and Behavioural Science, Auckland University Medical School, Aukland, New Zealand

Correspondence to: Rona Moss-Morris, Department of Psychiatry and Behavioural Science, Auckland University School of Medicine, Private Bag 92 019, Auckland, New Zealand.

1 Fukuda K, Straus SE, Hickie I, Sharpe MC, Dobbins JG, Komaroff A The chronic fatioue syndrome: tion and study. Ann Intern Med 1994;121:953-9.

2 Holmes GP, Kaplan JE, Gantz NM, et al. Chronic fatigue syndrome: working case definition. Ann Intern Med 1988;108:387-9.

3 Lezak MD. Neuropsychological assessment. 2nd ed. New York: Oxford University Press, 1983.

4 Altay HT, Toner BB, Brooker H, Abbey SE, Salit IE, Garfinkel PE. The neuropsychological dimensions of postinfectious neuromyasthenia (chronic fatigue syndrome): a preliminary report. Int $\mathcal{f}$ Psychiatry Med 1990;20:141-9.

5 Cope H, Pernet A, Kendall B, David A. Cognitive functioning and mag netic resonance imaging in chronic fatigue. $B r f$ Psychiatry 1995;167: 86-94.
6 Grafman J, Schwartz V, Dale JK, Scheffers M, Houser C, Straus SE. Analysis of neuropsychological functioning in patients with chronic fatigue syndrome. $\mp$ Neurol Neurosurg Psychiatry 1993;56:684-9.

7 Riccio M, Thompson C, Wilson B, Morgan DJR, Lant AF Neuropsychological and psychiatric abnormalities in myalgic encephalomyelitis: a preliminary report. $\mathrm{Br} \mathcal{f}$ Clin Psychol 1992;31: encephalom.

8 Scheffers MK, Johnson SK, Grafman J, Dale JK, Straus SE. Attention and short-term memory in chronic fatigue syndrome patients: an eventrelated potential analysis. Neurology $1992 ; 42: 1667-75$.

9 Prasher D, Smith A, Findley L. Sensory and cognitive event related potentials in myalgic encephalomyelitis. $\mathcal{F}$ Neurol Neurosurg Psychiatry 1990; 1:253-7.

10 Sandman CA, Barron, JL, Nackoul K, Goldstein J, Fidler F. Memory deficits associated with chronic fatigue immune dysfunction syndrome. Biol Psychiatry 1993;33:618-23.

11 Krupp LB, Sliwinski M, Masur DM, Freidberg F, Coyle PK. Cognitive functioning and depression in patients with chronic fatigue syndrome and multiple sclerosis. Arch Neurol 1994;51:705-10

12 Schmaling KB, DiClementi JD, Cullum M, Jones JF. Cognitive functioning in chronic fatigue syndrome and depression: a preliminary comparison. Psychol Med 1994;56:383-88.

13 Smith A. Cognitive changes in myalgic encephalomyelitis. In: Jenkins $\mathbf{R}$, Mowbray JF, eds. Post-viral fatigue syndrome. New York: Wiley, 1991: 179-94.

14 Smith AP, Behan PO, Bell W, Millar K, Bakheit M. Behavioural problem associated with chronic fatigue syndrome. $B r \mathcal{F}$ Psychol 1993;84:411-23.

15 McDonald E, Cope H, David A. Cognitive impairments in patients with chronic fatigue: a preliminary study. $f$ Neurol Neurosurg Psychiatry $1993 ; 56: 812-5$

16 Millon C, Salvato F, Blaney N, et al. A psychological assessment of chronic fatigue syndrome/chronic Epstein-Barr virus patients. Psychol Health 1989;31:31-41.

17 DeLuca J, Johnson SK, Beldowicz D, Natelson BH. Neuropsychological impairments in chronic fatigue syndrome, multiple sclerosis, and depression. F Neurol Neurosurg Psychiatry 1995;58:38-43.

18 DeLuca J, Johnson SK, Natelson BH. Information processing in chronic fatigue syndrome and multiple sclerosis. Arch Neurol 1993;50:301-4.

19 Ray C, Phillips L, Weir WRC. Quality of attention in chronic fatigue syndrome: subjective reports of everyday attention and cognitive difficulty and performance on tasks of focused attention. Br $\mathcal{f}$ Clin Psychol 1993;32:357-64.

20 Brickman AL, Fins AI. Psychological and cognitive aspects of chronic fatigue syndrome. In: Goodnick PJ, Klimas NA, eds. Chronic fatigue and related immune deficiency syndrome. Washington: American Psychiatric Press, 1993: 67-93.

21 Johnson SK, DeLuca J, Fiedler N, Natelson BH. Cognitive functioning in chronic fatigue syndrome. Clin Infect Dis 1994;18(suppl 1):S84-5.

22 Joyce E, Blumenthal S, Wessely S. Memory, attention and executive function in chronic fatigue syndrome. $\mathcal{F}$ Neurol Neurosurg Psychiatry 1996;60: in chronic

23 van Zomeren AH, Brouwer WH. Clinical neuropsychology of attention. New York: Oxford University Press, 1994.

24 Grant DA, Berg EA. A behavioral analysis of degree of reinforcement and ease of shifting to new responses in a Weigl-type card sorting problem. $\mathcal{f}$ Exp Psychol 1948;38:404-11.

25 Keefe RSE. The contribution of neuropsychology to psychiatry. Am Psychiatry 1995;152:6-15.

26 Katon WJ, Buchwald DS, Simon GE, Russo J, Mease PJ. Psychiatric illness in patients with chronic fatigue and rheumatoid arthritis. 7 Gen Intern Med 1991;6:277-85.

27 Wessely S, Powell R. Fatigue syndromes: a comparison of chronic "postviral" fatigue with neuromuscular and affective disorders. $f$ Neurol Neurosurg Psychiatry 1989;42:940-8.

28 Brand N, Jolles J. Information processing in depression and anxiety. Psychol Med 1987;17:145-55.

29 Cohen RM, Weingartner H, Smallberg SA, Pickar D, Murphy DL. Effort and cognition in depression. Arch Gen Psychiatry 1982;39:593-7.

30 Sternberg DE, Jarvick ME. Memory functions in depression: improvement with antidepressant medication. Arch Gen Psychiatry 1976;33: $219-24$

31 Ray C. Chronic fatigue syndrome and depression: conceptual and methodological ambiguities. Psychol Med 1991;21:1-9.

32 Wood GC, Bentall RP, Gopfert M, Dewey ME, Edwards RHT. The differential response of chronic fatigue, neurotic and muscular dystrophy patients to experimental psychological stress. Psychol Med 1994;24: 357-64.

33 Petrie K, Moss-Morris R, Weinman J. The impact of catastrophic beliefs on functioning in chronic fatigue syndrome. $\mathcal{f}$ Psychosom Research 1995;39:31-7. 